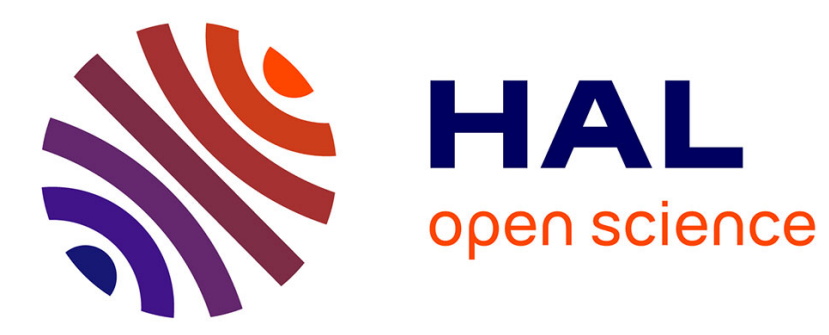

\title{
La tactilité d'une parole. Le pervers et la substance Houria Abdelouahed
}

\section{To cite this version:}

Houria Abdelouahed. La tactilité d'une parole. Le pervers et la substance. Cliniques méditerranéennes, 2005, Précarité, exclusion, abandon, 2 (72), pp.185 - 198. 10.3917/cm.072.0185 . hal01508559

\section{HAL Id: hal-01508559 \\ https://hal.science/hal-01508559}

Submitted on 19 Jun 2017

HAL is a multi-disciplinary open access archive for the deposit and dissemination of scientific research documents, whether they are published or not. The documents may come from teaching and research institutions in France or abroad, or from public or private research centers.
L'archive ouverte pluridisciplinaire HAL, est destinée au dépôt et à la diffusion de documents scientifiques de niveau recherche, publiés ou non, émanant des établissements d'enseignement et de recherche français ou étrangers, des laboratoires publics ou privés. 


\title{
Houriya Abdelouahed
}

\author{
La tactilité d'une parole \\ Le pervers et la substance
}

"De même que la psychose ne tient pas dans la seule éclosion d'une folie manifeste,

la perversion n'a nul besoin de devenir cruauté sadique, meurtre, viol ou toute autre monstruosité pour être repérée dans l'ordre du discours qu'elle compose » Pierre Fédida, 1977.

"La névrose est pour ainsi dire le négatif de la perversion ", écrivait Freud en 19051.

Si la névrose est le négatif de la perversion, c'est parce que le sexe ne peut s'entendre en dehors de l'assomption de la castration. Ce tournant décisif et structural qui signifie la rencontre avec l'instance tierce et l'accès à l'interdit de l'inceste en tant que loi. Un tel accès suppose un renoncement à l'objet primordial du désir et l'acquisition de la différence des sexes qui vient relayer le fantasme selon lequel tous les êtres sont pourvus d'un pénis. La symbolisation du manque qu'incarne la différence sexuelle ouvre sur la question du désir et de la filiation ainsi que sur celle du savoir (l'enfant découvre que son savoir était erroné et que le père en savait plus que lui quant au manque ${ }^{2}$ ). L'on comprend que l'acquisition de la différence sexuelle ne puisse se faire qu'au prix de grandes luttes internes. Mais le renoncement à l'objet et l'acceptation du manque comme cause du désir aboutissent à l'instauration et la mise en place d'une réalité psychique. Or, c'est justement cette « métamorphose » (P. Aulagnier) de l'enfant dans son rapport au désir et à

Houriya Abdelouahed, maître de conférences à l'université Paris 7 - Denis Diderot, psychanalyste, 83 rue Napoléon Fauveau, F-95170 Deuil La Barre.

1. S. Freud, Les trois essais sur la théorie de la sexualité, Paris, Gallimard, coll. «Idées », p. 54.

2. Cf. J. Clavreul, «Le couple pervers », dans Le désir et la perversion, Paris, Le Seuil, 1967, p. 93-126. 
son objet qui demeure problématique voire inaccessible au pervers, qui aura recourt au désaveu comme mode de défense face à l'angoisse de la castration. «Le processus était donc celui-ci : l'enfant s'était refusé à prendre connaissance de la réalité de sa perception : la femme ne possède pas de pénis ${ }^{3}$. "

Donc, le choc de la rencontre entre le regard et «l'effroi devant l'organe génital de la femme ${ }^{4} »-$ va mobiliser chez le pervers le désir de triompher sur la menace de la castration qui vient rappeler que ce qui est peut être perdu. Le pervers crée alors un substitut à ce pénis maternel « en vain cherché 5 » : le fétiche comme compromis entre « le poids de la perception non souhaitée et la force du contre-désir ${ }^{6} »$. Compromis qui vient témoigner de la domination des processus primaires. Le prix sera une déchirure intrasubjective, déchirure au sein du moi puisque «les deux positions, celle fondée sur le désir, et celle fondée sur la réalité, coexistent ${ }^{7}$. »

« Effroi devant l'organe génital de la femme » ou « horreur de la castration ", ces expressions insistent sur la rencontre entre le regard et le vu et posent la problématique scopique comme centrale. Seulement, la scène ne se ferme pas sur deux partenaires, une clôture entre le regard du garçon et le sexe de la femme. Un troisième terme fait son entrée, celui que P. Aulagnier nomme : Un regard Autre, « celui qui voit le sujet regardant 8 ». Un regard Autre qui soulève la question du désir de l'Autre. Et pour autant que cet Autre trouve son représentant initial dans la mère, l'on ne peut que faire nôtre le questionnement de J. Clavreul : « De quel œil la mère voit-elle son enfant qui la regarde? » Question centrale dans le travail analytique auprès d'un patient pervers. Si le désaveu du pervers porte sur le manque comme cause du désir et sur le manque de savoir comme cause de la pulsion scoptophilique ${ }^{9}$ (puisque le pervers refuse que son savoir soit précédé par un nonsu et que le père savait quelque chose que lui ignorait), le pervers ne manque pas de défier l'analyste comme sujet-supposé-savoir. Ce dernier sera confiné à cette place de simple moralisateur ou simple voyeur (ce qui ne manque pas de porter ainsi atteinte à la dissymétrie de la situation psychanalytique).

Poussant l'analyse de J. Clavreul, nous formulons les questions suivantes :

3. S. Freud, «Le fétichisme », dans La vie sexuelle, Paris, PUF, 1969, p. 134.

4. S. Freud, «Le clivage du moi dans les processus de défense », dans Résultats, idées, problèmes, II, Paris, PUF, 1985, p. 285.

5. Ibid.

6. S. Freud, «Le fétichisme», op. cit., p. 135.

7. Ibid., p. 137.

8. P. Aulagnier, Le désir et la perversion, op. cit., p. 46-47.

9. J. Clavreul, op. cit., p. 109. 
- le défi lancé par le pervers au sujet-supposé-savoir ne serait-il pas, en fait, un défi au père devant ce regard Autre?

- et si l'on faisait de la parole le véritable indice de la structure perverse (structure au-delà de telle ou telle modalité que prend l'acte pervers), ne serait-ce pas la parole qui permet de rendre compte de ce regard Autre : «Celui qui voit le sujet regardant»?

La parole du pervers - toute sa parole - dit le désaveu. Ce dernier s'ancre au plus profond de la structure langagière. Et celle-ci porte le sceau et la marque de ce qui caractérise la perversion : désaveu, transgression et jouissance. Tout le discours porte la marque indélébile d'une transgression qui jouit ainsi du désaveu. Et le discours qui dit raisonnablement l'excès et « la déraison du désir » (P. Aulagnier) est en fait ce qui vient témoigner de la continuité de la présence hallucinatoire de ce regard Autre.

\section{LE STYLE D'UNE PRÉSENCE}

Aimé. « Le trop aimé, le mal aimé ». Tel, dit-il, est « son drame ».

Avant de le rencontrer, l'assistante sociale chargée de lui trouver dans l'urgence un logement m'a parlé de lui. Comme il porte des sous-vêtements féminins, il refuse, dit-elle, de partager avec un autre homme une chambre dans un foyer d'urgence (ces foyers sont ouverts surtout en hiver). L'assistante sociale ajoute qu'il a une histoire trop difficile qui nécessite un suivi thérapeutique. Je consens à le recevoir au CMP.

D'une aventure thérapeutique, je garde le souvenir d'un malaise saisissant. Le doute porte sur le père. Il ne sait si son père est celui qu'il n'a jamais connu, l'ex-mari de la mère, ou si c'est son actuel compagnon ou quelqu'un $\mathrm{d}$ 'autre. Aimé a grandi auprès de sa sœur, son beau-père et sa mère, une mère aimant « le fouet » et « les scènes ». C'est tout ce qu'il peut dire de son histoire. Rapidement, le patient glisse de l'évocation d'un fétichisme vers la description minutieuse de sodomie incestueuse, description détaillée des scènes où les membres d'une même famille se livraient à des orgies sexuelles brutes. Minutieusement, il raconte comment il sodomisait sa sœur, détaillant ainsi les scènes avec un débit qui rend impossible toute ponctuation. Dès le début, je me suis sentie piégée par ce patient qui lançait un véritable défi au travail psychothérapeutique. Piégée ne serait-ce que parce que j'ai accepté de le recevoir avec l'assistante sociale chargée du suivi de son dossier ${ }^{10}$. Lors de cette

10. Il arrive que les thérapeutes reçoivent, lors d'un premier entretien, un patient avec une tierce personne. Je ne pourrai traiter de la question du cadre ici car cela m'amènerait à un autre problème clinique et théorique qui demanderait un long développement que je réserve pour une étude prochaine. 
première rencontre remplie d'histoires de fouet et de sodomie, après que je lui ai signifié la fin de la séance, il dit qu'il veut absolument remercier l'assistante sociale de s'être dévouée, qu'il tient à lui dire combien il l'aime et il continue en regardant l'assistante sociale : "Je vous aime comme ma sœur ${ }^{11}$. » La sidération empêcha toute parole.

Les séances se suivaient, mais de sa vie il ne disait rien. Il m'est difficile aujourd'hui de restituer son histoire, de tracer l'anamnèse. Tout est englouti dans la description des scènes sexuelles de famille. Ces descriptions prenaient dans sa parole une telle intensité qu'il me semblait voir. J'étais, en effet, réduite à un simple spectateur des scènes perverses. Plus que cela, la parole d'Aimé décrivant un magma pulsionnel, engloutissant jusqu'à la trace minime de l'écart, était intensément visqueuse. La viscosité de cette parole me touchait telle une substance gluante. Et sa tactilité non seulement attaquait ma capacité de liaison, mais abolissait distance et frontière. La tactilité de cette parole, annulant tout écart, détruisait ce qui définit la parole comme parole. La parole d'Aimé était soustraite à la puissance de l'interdit, interdit de toucher. Or, " l'attouchement, écrit Freud, est le commencement de toute tentative de s'emparer d'une personne ou d'une chose, de l'assujettir, d'en tirer des services exclusifs et personnels $12 »$.

Même s'il côtoie la psychose, le pervers n'y sombre pas car du moment où il reconstitue ailleurs le champ de l'illusion, cet ailleurs étant le fétiche, son savoir, comme le rappelle J. Clavreul, est non absolu. Nonobstant, c'est la structure langagière elle-même qui porte les marques d'une telle implantation. La visée du fétichiste - et c'est toute la singularité de sa position - est de garantir l'Autre incastrable, soit de "produire le phallus en tant que tel ${ }^{13} »$ dans le langage.

Contrairement au psychotique pris dans le déchaînement du signifiant, le pervers a un langage logiquement structuré. Toutefois, cette logique langagière est ce qui vient - dans la parole du pervers - dire « la déraison du désir » selon l'expression de P. Aulagnier qui écrit justement : « Le pervers est celui qui parle raisonnablement, génialement parfois -, de la déraison du désir ${ }^{14}$. »

11. J'ai appris plus tard qu'il demandait à l'Espace territorial de prendre en charge du point de vue financier le projet de son entreprise de lingerie féminine. Il se présenta également à la mission locale avec le projet de cadrer les jeunes. Ses projets tentent de lier des instances sociales et juridiques à sa structure psychopathologique. On peut dire également que par ces différents projets, il tente de triompher de la loi ; ne serait-ce que parce qu'il demande aux instances sociales et juridiques (Les Espaces territoriaux travaillent avec des représentants de la préfecture) de cautionner son fonctionnement.

12. S. Freud, Totem et tabou, PBP, p. 45.

13. H. Rey-Flaud, Comment Freud inventa le fétichisme... et réinventa la psychanalyse, Paris, Payot, 1994, p. 192-193.

14. P. Aulagnier, « La perversion comme structure », L'inconscient, $\mathrm{n}^{\circ}$ 2, avril-juin, p. 14. 
R. Barthes a bien repéré cette idée de l'ordre dans la pratique sadienne. Les dérèglements sont énergiquement réglés. Si la luxure est sans frein, notet-il, elle n'est pas sans ordre. «Un moment, dit Delbène, tout en feu, un instant, mes bonnes amies, mettons un peu d'ordre à nos plaisirs, on n'en jouit qu'en les fixant » (cité par R. Barthes). Le pervers introduit de l'ordre non seulement dans les pratiques mais dans le langage qui dit et décrit de telles pratiques. Ainsi, « hors le meurtre, il n'y a qu'un trait que les libertins possèdent en propre et ne partagent jamais, sous quelque forme que ce soit : c'est la parole. Le maître est celui qui parle, qui dispose du langage dans son entier $15 »$.

Passé la scène, on retrouve, écrit encore R. Barthes, le récit ou la dissertation. Laquelle dissertation, apparemment bien structurée et raisonnable, se confond entièrement avec l'énergie du vice qui contamine tous les styles du discours. " le narratif, le lyrique, le moral, la maxime, le topo mythologique 16 ». Et R. Barthes de continuer : "Nous commençons à savoir que les transgressions du langage possèdent un pouvoir d'offense au moins aussi fort que celui des transgressions morales ${ }^{17}$. » L'univers du pervers devient un univers du discours et la pratique suit la parole et « en reçoit absolument sa détermination : ce qui se fait a été dit 18 ». La parole devient ainsi le seul drame. C'est une parole qui débouche sur « un condensé d'inceste ». Et R. Barthes de continuer : «C'est-à-dire sur un sens ${ }^{19}$. "

Là se trouve justement le cynisme du pervers : rester dans des formes articulatoires structurées pour dire " la déraison du désir » et son excès. Ainsi, même si le code érotique bénéficie de la logique du langage, il n'empêche que le langage n'est plus capacité de nomination de par une tactilité qui efface et abolit toute séparation annulant ainsi ce qui définit la parole comme parole.

Avec Sade et Masoch, la littérature sert à dire (et non à nommer) non pas « le monde puisque c'est déjà fait, mais une sorte de double monde, capable $\mathrm{d}^{\prime}$ en recueillir la violence et l'excès ${ }^{20}$ » parvenant ainsi à établir celui que l'on nomme l'Être suprême en méchanceté dans un dépassement de la loi et le rétablissement d'une «nature première », selon l'expression de G. Deleuze ${ }^{21}$.

15. R. Barthes, Sade, Fourier, Loyola, Paris, Le Seuil, coll. « Essais », 1971, p. 34.

16. Op. cit., p. 34 .

17. Op. cit., p. 37.

18. Op. cit., p. 38.

19. Op. cit., p. 36.

20. G. Deleuze, « Présentation de Sacher-Masoch », dans La Vénus à la fourrure, Éd. de Minuit, 1967, p. 33.

21. Op. cit., p. 76-77. 
Si le névrosé fait appel à la négation comme forme voilée de la parole inconsciente, le pervers, lui, recourt au désaveu qui lui permette de présenter une nomenclature érotique nue. Et si l'on se souvient de ce qu'écrivait Lacan sur « l'impudeur de l'un » faisant « le viol de la pudeur de l'autre 22 », l'on peut dire que le viol de la pudeur émane du fait d'être au contact de ce qui doit rester voilé et qui se trouve mis à nu par le discours du pervers. La nudité est celle de la nature première. En cela, le pervers effectue un coup de force contre le langage.

Le travail d'Henri Rey-Flaud offre de bonnes assises théoriques pour réfléchir sur ce " coup de force » entrepris contre l'Autre en tant que tel. Le pervers, écrit-il, vise à faire passer sous le contrôle du sujet « la barre qui, marquant l'arbitraire du signe, constitue l'essence du langage représentatif 23 ».

Dans son désaveu de la castration et sa tentative d'esquiver l'abîme menaçant de la psychose, le pervers tente de contrôler le langage au point précis où s'établit sa garantie. " Le pervers effectue ce forçage au moment où en "hallucinant le phallus", il produit en lieu et place du signe arbitraire un signe motivé soumis à sa volonté. Cette opération est bien une entreprise de subversion du langage, puisqu'elle réalise une représentation "forcée" au lieu où se perd normalement le "représentant" (non représentatif) de la représentation [...] Par ce coup de force le fétichiste, en s'emparant du signifiant qui est au fondement du système signifiant, s'assure le contrôle de ce système et se garde, du même coup, de la psychose ${ }^{24}$. " Ainsi, le fétichiste appelle le fétiche, cet objet paradoxal, à remplir la fonction d'incarner «le signifiant du manque dans l'Autre ». Le désaveu pervers porte ainsi sur le "signifiant du manque dans l'Autre ». Et c'est en cela précisément que demeure le coup de force du pervers contre le langage. Coup de force car le désaveu se situe au niveau logique du fondement du langage.

Se saisissant de la formule d'Octave Mannoni : « Je sais bien mais quand même », H. Rey-Flaud exprime ainsi le clivage du moi chez le fétichiste : «Je sais bien qu'un pied est un pied mais quand même... »Ce qui fait la singularité irréductible du fétichiste, c'est qu'en voyant l'objet, il voit également la Chose ${ }^{25}$. Le désaveu et le clivage du moi lui permettent d'accéder à la chose dans sa nudité.

Ici, la nudité est celle de la substance.

22. J. Lacan, «Kant avec Sade », dans Écrits, Paris, Le Seuil, 1966, p. 790.

23. H. Rey-Flaud, op. cit., p. 298.

24. Ibidem.

25. H. Rey-Flaud, op. cit., p. 100. 


\section{LA SUBSTANCE ET L'INCESTE}

Aristote définit la substance comme « la chose première » ou « la matière première absolument indéterminée ${ }^{26}$ ».

La pensée philosophique accorde une large place à la question de la substance ${ }^{27}$. Mais dans le cadre de ce travail et afin de réfléchir sur la substance dans la parole, nous allons nous pencher sur l'œuvre de F. Héritier.

Il revient, en effet, à F. Héritier le mérite, dans une réflexion sur ce qu'elle nomme " un inceste de second type », de concevoir la prohibition de l'inceste comme un problème de "mécanique des fluides 28 ». Ce qui fonde la prohibition, dit-elle, c'est le passage des substances d'un corps à un autre. L'interdit porte sur " le cumul de l'identique " ou "le contact des identités », à savoir « les identités substantielles ». La mise en contact « d'humeurs identiques », continue F. Héritier, met en jeu ce qu'il y a de plus fondamental dans les sociétés humaines à savoir la façon dont elles conçoivent et bâtissent leur catégories de l'identique et du différent comme " catégories princeps » de la pensée.

La parole d'Aimé a le pouvoir de toucher par la substance. Et la tactilité de cette parole engloutit jusqu'à la trace de l'écart. Ce qui se trouve annulé, c'est la juste distance des corps, ce qui se trouve aboli, c'est le différent comme catégorie de la pensée. Ainsi, plus qu'un scénario exhibitionniste livré par Aimé, c'est la thématique orale qui invalide le lieu de séance. L'impudeur du pervers consiste en ce geste de dévoilement, au sein de la parole, de la substance. Ici, incestueuse. Si l'on ne peut que suivre R. Barthes rappelant que la parole constitue, dans la perversion, le seul drame car elle débouche sur un "condensé d'inceste ", il n'empêche que ce dernier n'équivaut pas, comme l'écrit R. Barthes, "au sens 29 », mais à ce qui l'annule. Le sens ne se constitue que de la séparation d'avec l'inceste.

Avec un débit haletant, Aimé raconte. Le silence étant répudié, sa parole devient orgie. La bouche devient organe sexuel. Organe sexuel car « la tentative orale de la parole » (P. Fédida) ne se soutient pas du négatif d'une rencontre, mais se nourrit du contact. C'est une parole qui touche. Le cynisme du pervers réside-t-il, alors, dans cette jouissance de voir l'horreur de celui qui est touché par la substance ? Toucher qui «présuppose, comme l'écrit Freud, nécessairement le toucher corporel, l'action de porter la main ${ }^{30} »$. Ici,

26. Aristote, « Livre $Z$ », La métaphysique, Librairie philosophique Vrin, 1991, p. 355.

27. Cf. A. Lalande, Vocabulaire technique et critique de la philosophie, Paris, PUF, Quadrige, 2002.

28. F. Héritier, Les deux sœurs et leur mère, Paris, Odile Jacob, 1995, coll. « Opus ».

29. R. Barthes, op. cit., p. 36.

30. S. Freud, Inhibition, symptôme et angoisse, Paris, PUF, 1990, p. 37. 
action de porter la main depuis la bouche. "L'usage de la bouche comme organe sexuel est considéré comme perversion », dit Freud ${ }^{31}$. Avec la main, la bouche constitue justement dans la perversion « la totalité du terrain expérimental ${ }^{32}$ ».

\section{D'UNE ORALITÉ VORACE}

Si l'athéisme de Sade est scélérat, c'est parce qu'il veut instituer au cœur même de " l'acte suprême » de la raison normative « le règne de l'absence totale des normes $33 »$. Tout en respectant les normes de la structure discursive de l'espèce humaine, Sade veut établir une contre-généralité valant cette fois pour la spécificité des perversions. Là demeure « la notion sadienne de monstruosité intégrale ». P. Klossowski a tout à fait raison de parler de l'insubordination «fonctionnelle » qui vient désorganiser la raison normative. Parler raisonnablement de "la déraison du désir » est le coup de force du pervers. Et là où le psychotique est pris dans le déchaînement du signifiant, le pervers entreprend son coup de force contre le langage. Là où Wolfson tente de " délimiter de façon folle les arpents des différentes langues ${ }^{34}$ », le pervers - tout en disant l'excès et la « déraison du désir » - respecte la structure discursive.

Le rapport à l'oralité n'est pas le même. Dans sa lutte contre la mère ${ }^{35}$, Wolfson parle d'orgies, du gouffre de l'oral et de l'avidité du gloutonnement. L'horreur du gavage va de pair avec cette nécessité de détruire et construire de façon folle le matériau linguistique ${ }^{36}$. Wolfson tue la langue maternelle, en « démembrant » et en « désossant » les mots anglais de la langue maternelle qu'il remplace par des mots étrangers de sens identique et ayant des phonèmes communs. Le résultat sera " un pot pourri de divers idiomes ». Et le « cannibalisme linguistique » (R. Gori) servira de " moyen bizarre, artifi-

31. S. Freud, Les trois essais sur la théorie de la sexualité, p. 36.

32. M. Khan, Figures de la perversion, Paris, Gallimard, 1981, p. 227.

33. P. Klossowski, Sade mon prochain, Paris, Le Seuil, coll. «Essais », 1967, p. 19.

34. P. Aulagnier, Un interprète en quête de sens, Paris, Payot, 1991, 294.

35. Contre l'œil en moins de la mère de L. Wolfson, est l'œil complice (en trop ?) de la mère regardant son enfant à un tournant décisif de son histoire.

36. "Ces jours-là, dans sa faiblesse et dans sa faim, il perdait non rarement la tête, commençant alors dans son délire ou dans sa démence une vraie orgie - ouvrant de nouveaux contenants l'un après l'autre et toujours mangeant voracement, compulsivement le tout jusqu'au dernier morceau [...] Je nourrissais toute la sainte journée l'intention de ne manger qu'un petit peu et voilà j'ai fait dans une sacrée heure le contraire de ce que je projetais durant des heures, des heures et des heures! Hélas ! je suppose, je suppose que cela prouve que j'ai l'esprit borné, que je suis incapable d'apprendre toutes ces langues dans l'étude desquelles j'ai mis tellement de temps. O merde ! » L. Wolfson, Le schizo et les langues, Paris, Gallimard, 1970, p. 48, p. 50. 
ciel, contre nature » (Wolfson). Car aucune règle syntaxique, comme le fait remarquer G. Deleuze, ne vient définir cet ensemble en y faisant correspondre les sens aux sons ${ }^{37}$ afin de détruire la langue maternelle. La destruction de celle-ci implique une décomposition phonétique du mot. Laquelle décomposition se fait dans un magma réunissant toutes les langues contre la langue maternelle. " Les nourritures sont à leur tour des morceaux organiques, dont chacun à lui-même un morceau de sein, un morceau d'excrément, un morceau d'enfant, un morceau de pénis, larves nombreuses. Et le rapport des deux sortes de morceaux, verbaux et organiques, n'est pas de désignation ni d'expression, mais d'imbrication violente, les uns dans les autres, les uns sur les autres ${ }^{38}$. »

Seulement si le langage subit ici la subversion de la pulsion de mort, la dissection n'en est pas moins une tentative de se constituer, passant par les langues, un après-coup. Le savoir sur les langues, comme l'a bien fait remarquer $\mathrm{P}$. Aulagnier, est le chemin à parcourir afin que naisse le Je de l'énoncé ${ }^{39}$. En cela, le cannibalisme de Wolfson diffère de la voracité d'Aimé. Celle-ci dévore la distance chez l'autre pour le maîtriser. Aussi l'annulation de l'écart a-t-elle pour effet une désubjectivation chez celui qui est pris dans les filets d'une parole ne livrant que l'excès.

Si la psychose se signe de l'exclusion d' « un dedans primitif » qui est le premier corps du signifiant (J. Lacan) mettant à nu « le palimpseste de l'imaginaire » et conférant à la psychose le qualificatif d'un fonctionnement à ciel ouvert, la perversion se nourrit, elle, d'une clôture. Clôture qui permet le système. Et la volupté d'être va de pair avec cette clôture ${ }^{40}$. Ajoutons que cette dernière trouve asile dans la barricade d'une logique langagière qui se nourrit du désaveu. Un désaveu qui s'ancre au plus profond de la structure discursive et qui témoigne néanmoins de la transgression. Mais, non pas transgression de telle ou telle conduite ou norme sociale, mais transgression de ce par quoi s'énonce la loi, à savoir le langage. La transgression du pervers devient loi. La psychose est une tentative de guérison. La perversion, quant à elle, est une tentative d'esquiver le gouffre de la psychose.

37. G. Deleuze, « Préface » à Le schizo et les langues, op. cit., p. 9.

38. Op. cit., p. 16.

39. Comme chez Fritz Zorn, le temps de l'écriture est temps de construction de l'après-coup et du sens.

40. R. Barthe, op. cit., p. 20-21. 


\section{LA MÈRE ORALE}

Dans sa lecture de La Vénus à la fourrure, G. Deleuze dégage trois mères qui correspondent aux images fondamentales de mère : "La mère primitive, utérine, hétaïrique, mère des cloaques et des marais - la mère œedipienne, image de l'amante, celle qui entrera en rapport avec le père sadique, soit comme victime soit comme complice - mais entre les deux, la mère orale, mère des steppes et grande nourrice, porteuse de mort ${ }^{41}$. » Ces trois femmes désignent moins trois personnages différents et juxtaposés que trois moments de la femme dans une fantasmatique ou «trois points cardinaux de l'organisation perverse $42 »$. Toutes les fonctions maternelles seront, dans le masochisme, condensées sur la seconde mère, « la mère orale » qui seral'élément propre de la perversion masochiste. Cette concentration sur la mère orale implique le premier aspect d'après lequel le père est annulé. " Les femmes constituent un ordre symbolique dans lequel ou par lequel le père est déjà supprimé, supprimé de tout temps ${ }^{43}$. »

À la « dénégation magnifiante de la mère ("Non, la mère ne manque symboliquement de rien"), correspond une dénégation annulante du père ("le père n'est rien"), c'est-à-dire qu'il est privé de toute fonction symbolique ». La dénégation fétichiste ( «la mère ne manque pas de phallus ») n'est pas une forme de dénégation comme un autre ou parmi d'autres. C'est le principe d'où dérivent toutes les autres figures, «l'annulation du père et le désaveu de la sexualité » 44 .

La mère orale affirme « la totalité et l'autonomie de ses pouvoirs. En ce sens, elle réalise une image composée de la mère non assujettie à un désir et capable de répondre au désir du sujet. Cette mère détient la loi et recueille en elle l'amour que le sujet lui porte, ceci rend compte alors d'une possibilité d'inceste ». La loi détenue par la mère suppose l'annulation préalable du principe du tiers séparateur dont le fondement est justement l'interdiction de l'inceste. À ce moment, l'on peut dire à l'instar de P. Fédida qu'au cœur de la structure perverse, demeure le triomphe de la mère orale. Celle-ci serait « le principe maternel à l'état pur 45 ». Ainsi, « La glorieuse mère orale 46 » devient-elle la maîtresse de la loi.

41. G. Deleuze, «Présentation de Sacher-Masoch », dans La Vénus à la fourrure, Éd. de Minuit, 1967, p. 49.

42. P. Fédida, Le concept et la violence, 10-18, 1977, p. 35.

43. G. Deleuze, op. cit., p. 55 et 56.

44. Op. cit., p. $56-57$ et p. 109.

45. P. Fédida, op. cit., p. 39.

46. G. Deleuze, op. cit., p. 83. 
Le père est exclu, annulé. La loi n'étant plus fondée dans le père (en tant que principe séparateur), son maintien devient tout à fait formel de par le triomphe de la mère orale. Ainsi, lorsque P. Fédida désigne, dans la structure perverse, un point de vue qui assure " la sécurité et la cohérence du fantasme 47 ", nous pouvons ajouter que ce point de vue n'est autre que ce regard Autre, depuis une parole qui témoigne du triomphe de la mère orale. Par sa parole, le pervers laisse voir le triomphe de la mère orale dans son annulation du principe paternel.

Contrairement au langage psychotique, le langage du pervers respecte ordre et syntaxe. Mais c'est un langage qui touche par la chose première. La jouissance à ce moment n'est-elle pas celle de la mère orale rendant possible dans la parole du pervers un tel toucher ? La parole, écrit Freud dans Le mot d'esprit, prend son origine dans une action qui n'a pas lieu. La violence du pervers, son « impudeur », ne réside-t-elle pas dans l'annulation de la magie du mot ? La parole a une origine pulsionnelle et un ancrage corporel. Mais parce que le désir se fait parole, la satisfaction devient substitutive. La parole d'Aimé qui échappe à la puissance de l'interdit laisse voir que le plaisir n'est nullement métaphorique ni la satisfaction substitutive. Le condensé d'inceste ne débouche pas, comme l'écrit $R$. Barthes, sur un sens, mais sur l'éclatement du sens. Le coup de force et «le cynisme du pervers », coup de force cynique consiste en ceci : pervertir ce par quoi s'énonce la loi, faire du langage le discours raisonnable de la déraison. Subterfuge ingénieusement créé par le pervers contre ce qui fixe la structure psychique. Qu'il se serve d'un langage structuré pour énoncer la défaillance de ce qui soutient la structure langagière : l'interdit d'inceste. Ce n'est point la puissance nominative qui, à ce moment, définit le langage mais « l'énergie du vice ». L'outrage devient ce à partir de quoi se restructure le langage. Ce que P. Klossowski exprime de la façon suivante : «Ce que d'une part le langage logique en tant que celui de la raison vient adapter au geste codé du pervers, c'est l'athéisme, en tant

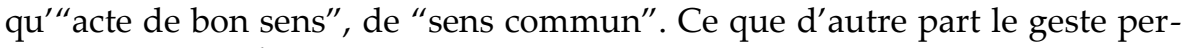
vers ainsi codifié introduit dans le langage du "sens commun", c'est le nonlangage de la monstruosité qui subsiste sous ce code. » « Le geste singulier $\mathrm{du}$ pervers, continue P. Klossowski vide d'un coup tout contenu de parole, puisqu'il est à lui seul tout le fait d'exister ${ }^{48}$. » Le langage logiquement structuré de Sade devient le terrain de l'outrage. Ce dernier (sodomie, inceste, aberration...) devient ce à partir de quoi se dit la parole et se restructure le langage. R. Barthes écrit que Sade inverse la maxime freudienne lorsqu'il fait

47. P. Fédida, op. cit., p. 40.

48. P. Klossowski, op. cit., p. 33 et p. 34 . 
venir le sperme à la place du mot. En fait, le sperme fait corps avec le mot. Le mot est taché de sperme. Le mot touche, colle, tache. À ce moment, ce qui est exalté, ce n'est point la magie du mot, mais la substance dénudée.

\section{CONCLUSION}

Le langage traîne avec lui une histoire d'amour et de sexe, mais il y a différentes façons de l'approcher. Celle de Francis Ponge - où l'amour des mots dit l'amour des choses -, celle du schizo, l'étudiant des langues, où la sexualité devient une pauvre chose «à l'état emboîté »(G. Deleuze), celle enfin du pervers où la sexualité se trouve bannie, annulée. Car la sexualité va de pair avec l'assomption de la castration. Cette dernière étant par définition le négatif qui fonde la parole comme parole.

L'essence linguistique de l'homme, écrit W. Benjamin, consiste en ce qu'il nomme les choses. "C'est donc en nommant toutes les autres choses que l'homme communique (autant qu'elle est communicable) sa propre essence spirituelle ${ }^{49}$. » Cette essence spirituelle est liée à l'œuvre de nomination. En effet, le nom est ce qui sort la forme du chaos originel, de l'indistinction confuse et obscure, de l'indifférenciation opaque. Dans le nom, l'essence spirituelle qui se communique " est le langage ", écrit encore W. Benjamin. Depuis la verticalité du souffle, chaque forme (nominative) devient « ange, astre ou encore cheval » (Ibn Arabi) ou pré (F. Ponge). Nominative est cette parole du moment où elle accepte le négatif qui en est le fond et le fondement. Nominative est la parole lorsqu'elle se soutient de la séparation, de l'absence et du manque. En venant à la place et au lieu du vide de la bouche, la parole tisse l'écart qui la fonde comme nominative. C'est sur un fond d'absence que naît le langage. En fondant, donc, le désir sur un fond de présence, le pervers effectue, en effet, un coup de force contre le langage. Il n'y a pas de forclusion dans la perversion, non plus de refoulement mais désaveu, un désaveu au cœur de la structure langagière. G. Deleuze a raison d'écrire que le modèle du pervers est plutôt dans " les institutions anarchiques ». Et quelle anarchie est plus forte que celle de faire du langage (régi par la loi) la révolution permanente du «non-langage »? Anarchiste, le pervers est-il pour autant maître du langage ? Son cynisme bute sur ceci : à savoir que si l'homme est le maître de la nature parce qu'il nomme les choses, il n'est nullement maître du langage.

49. W. Benjamin, Mythe et violence, I, Denoël, 1971, p. 82. 


\section{BIBLIOGRAPHIE}

Abdelouahed, H. 1996. «Des noms qui regardent », Cliniques méditerranéennes, $\mathrm{n}^{\circ} 51 / 52$, p. $145-155$.

Abdelouahed, H. 1998. La visualité du langage, Paris, L'Harmattan.

ARISTOTE. 1991. «Livre Z », dans La métaphysique, Librairie philosophique Vrin, p. 347452.

Aulagnier, P. 1991. "Le sens perdu ou le "schizo" et la signification », dans Un interprète en quête de sens, Payot, p. 286-316.

Aulagnier, P. «La perversion comme structure », L'inconscient, $\mathrm{n}^{\circ}$ 2, avril-juin, p. 1141.

BArthes, R. 1971. Sade, Fourier, Loyola, Paris, Le Seuil, coll. «Essais ».

Benjamin, W. 1971. "Sur le langage en général et sur le langage humain », dans Mythe et violence, I, Denoël, p. 71-98.

Clavreul, J. 1967. «Le couple pervers », dans Le désir et la perversion, Paris, Le Seuil, p. 91-117.

Deleuze, G. « Présentation de Sacher-Masoch », dans Sacher-Masoch, La Vénus à la fourrure, Paris, Éd. de Minuit, p. 13-115.

Deleuze, G. 1970. «Schizologie », dans Wolfson L. (sous la direction de), Le schizo et les langues, Paris, Gallimard, p. 5-23.

FÉDIDA, P. 1977. Le concept et la violence, Union générale d'éditions, 10/18.

Freud, S. 1905. Les trois essais sur la théorie de la sexualité, Paris, Gallimard, 1962.

FREUD, S. 1912. Totem et tabou, PBP.

FREUD, S. 1926. Inhibition, symptôme et angoisse, Paris, PUF, 1990.

FREUD, S. 1927. " Le fétichisme », dans La vie sexuelle, PUF, 1969, p. 133-138.

FreUd, S. 1938. « Le clivage du moi dans le processus de défense », dans Résultats, idées, problèmes, II, Paris, PUF, 1985, p. 283-286.

KHAN, M. 1981. Figures de la perversion, Paris, Gallimard.

KLOSsOWSKI, P. 1967. Sade mon prochain, Paris, Le Seuil, coll. « Essais ».

LACAN, J. 1966. « Kant avec Sade », dans Écrits, Paris, Le Seuil, p. 765-790.

LALANDE, A. 2002. Vocabulaire technique et critique de la philosophie, PUF/Quadrige.

Rey-Flaud, H. 1994. Comment Freud inventa le fétichisme... et réinventa la psychanalyse, Paris, Payot.

Rosolato, G. 1967. « Le fétichisme », dans Le désir et la perversion, Paris, Le Seuil, p. 752.

WOLFSON, L. 1970. Le schizo et les langues, Paris, Gallimard.

\section{Résumé}

Si le névrosé fait appel à la négation comme forme voilée de la parole inconsciente, le pervers recourt au désaveu qui lui permet de présenter une nomenclature érotique nue. Cette nudité est celle de la substance dans la parole du pervers, une substance qui touche. La tactilité de cette parole annule la distance détruisant ainsi ce qui fonde la parole comme parole. La parole du pervers atteste de l'exclusion du père en tant 
que principe séparateur au profit de la " glorieuse mère orale ». Et si des auteurs ont attiré l'attention sur le fait que le pervers parle raisonnablement de l'excès et de « la déraison du désir », il faut ajouter que toute sa logique discursive est ce qui sert à dire la défaillance de ce qui soutient la structure langagière, à savoir l'interdit d'inceste. C'est la sexualité qui se trouve ainsi bannie car cette dernière va de pair avec l'assomption de la castration en tant que négatif au fond et fondement de toute parole.

\section{Mots-clés}

Structure perverse, désaveu, castration, nudité, substance, oralité, parole, structure langagière, sexualité, interdit d'inceste.

THE TOUCH OF SPEECH. THE PERVERSE AND SUBSTANCE.

\section{Summary}

If the neurotic appeals to the act of denying as a veiled form of the inconscious speech, the pervert resorts to the act of retraction, which enables him to present as naked erotic nomenclature. This nakedness characterizes the substance of his speech, which turns out to be a touching substance. This tactile dimension suppresses any distance, thus destroying the very foundation of speech. The pervert's speech bears witness to the exclusion of the father as a separating principle, for the benefit of the "glorious oral mother ». Some authors have highlighted the pervert's reasonable speech about excess and about the " unreasonableness of desire ». Yet it should also be stated that the whole logics of his speech refers to the lapse of what holds up language structure, namely the prohibition of incest. Sexuality is therefore bannished, since it implies having assumed that castration is the negativity which lies under and founds any act of speech.

\section{Keywords}

Perverse structure, retraction, castration, nakedness, substance, orality, speech, language structure, sexuality, prohibition of incest. 